\title{
Patient-centeredness and empathy in a bilingual interprofessional primary care teaching clinic: a pilot study
}

This article was published in the following Dove Press journal:

Journal of Multidisciplinary Healthcare

23 August 2016

Number of times this article has been viewed

\author{
Sallie D Mayer' \\ Erika Peterfy ${ }^{2}$ \\ Steven H Crossman ${ }^{3}$ \\ Lisa Burroughs Phipps ${ }^{4}$ \\ Allison A Vanderbilt ${ }^{5}$ \\ 'Bon Secours Virginia Health \\ System, Bon Secours Medical Group, \\ Midlothian, VA, ${ }^{2}$ School of Pharmacy, \\ Virginia Commonwealth University, \\ Charlotte, NC, ${ }^{3}$ Department of Family \\ Medicine and Population Health, \\ College of Medicine, ${ }^{4}$ Department \\ of Pharmacotherapy and Outcomes \\ Science, School of Pharmacy, Virginia \\ Commonwealth University, Richmond, \\ VA, ${ }^{5}$ Department of Family Medicine, \\ College of Medicine and Life Sciences, \\ University of Toledo, Toledo, OH, USA
}

Correspondence: Sallie D Mayer Bon Secours Virginia Health System, Bon Secours Medical Group, 14366 Sommerville Court, Midlothian, VA 23II3, USA

Tel +l 8043379654

$\mathrm{Fax}+18043780938$

Email Sallie_Mayer@bshsi.org

\begin{abstract}
Utilizing the Consultation and Relational Empathy survey, this project examined the perceptions of care team empathy and patient-centeredness between English- and Spanishspeaking patients. From fall through spring semesters, patient surveys from a primary care, interprofessional student-led teaching clinic were collected and analyzed. Overall, mean scores for both English- and Spanish-speaking patients were above the reported normative average for general practitioners. While, overall, patients expressed satisfaction with the student-led teaching clinic in terms of empathy and patient-centeredness, English-speaking patients had higher median scores than Spanish-speaking patients. Analyzed individually, questions related to communication and provider attitudes were scored lower by Spanish-speaking patients. These results demonstrate that student-led clinics can deliver patient-centered care and highlight the continuing need to investigate and address disparities between English- and Spanish-speaking patients with regard to feelings of empathy and patient-centeredness.
\end{abstract}

Keywords: patient-centeredness, interprofessional care, uninsured patients, free clinics, student teams

\section{Introduction}

Medical schools across the US have established student-led free clinics to serve the poor and uninsured. A study performed by Simpson and Long in 2007 reported that 49 medical schools have at least one student-led clinic, representing 36,000 patientphysician visits annually. ${ }^{1}$ Such clinics serve as a safety net for poor and uninsured patients within an increasingly complex health care system. Patients benefit from access to medical providers, diagnostic tests, laboratory services, and prescription medications that would otherwise be unavailable.

In addition to providing clinical services to low-income and underserved patients, student-led clinics provide educational opportunities for all health profession students via interprofessional collaboration across the continuum of health care to better treat the patient. In these settings, students learn skills such as performing a physical examination, taking patient history, and how to present a patient to a supervising clinician. ${ }^{1}$ Students gain clinical experience through working with diverse patient populations while also attaining feelings of self-worth, medical humanism, and professional generosity. ${ }^{1,2}$ These clinical encounters further provide students the opportunity to improve cross-cultural communication skills through interactions with patients from diverse socioeconomic, racial, and ethnic backgrounds. ${ }^{2}$ Furthermore, these learning opportunities provide the students with the foundation for interprofessional collaboration 
and working in teams to care for complicated patients and confounding variables to assure better patient outcomes.

Studies have focused on the characteristics, clinical outcomes, and educational benefits of student-led clinics; none have examined patient satisfaction within an interprofessional setting. ${ }^{2,3}$ Multiple studies in different health care settings have demonstrated that Hispanic and Spanish-speaking patients experience lower satisfaction with their health care when compared to Whites and native English speakers. ${ }^{4-8}$ Spanishspeaking patients comprise the fast-growing subpopulation of US, and $\sim 30 \%$ of the patient population served by student-led clinics; therefore, it is increasingly important to focus on appropriate care and patient satisfaction within this population. ${ }^{1,7}$

Crucial elements of patient satisfaction and quality of care include patient-centeredness and provider empathy. Patientcenteredness is defined as providing care that is respectful of and responsive to individual patient preferences, needs, and values, and that ensures patient values guide all clinical decisions. ${ }^{9}$ Closely related to patient-centeredness is provider empathy, or the ability to understand the patient's situation, perspective, and feelings; to communicate that understanding and check its accuracy; and to act on that understanding with the patient in a helpful way. ${ }^{10}$ Provider empathy is a learnable skill that is crucial to developing a provider-patient relationship and is included as an educational objective in health professions training. Provider empathy has been shown to increase diagnostic accuracy, patient participation, patient adherence, and patient satisfaction. ${ }^{6,10}$

Utilizing patient surveys from an outpatient, student-led teaching clinic for medical, pharmacy, and medical interpreter students, the first aim of this project was to compare patient perceptions of care team empathy and patient-centeredness to normative values. The second aim of this study was to identify whether differences exist in satisfaction and feelings of patient-centeredness between English- and Spanish-speaking patients in this student-led interprofessional teaching clinic. Our hypothesis was "With specific training, the interprofessional care team will provide patient-centered care that was equally satisfactory to all patients served in the student-led interprofessional teaching clinic."

\section{Methods}

\section{Instrument}

The Consultation and Relational Empathy (CARE) survey is a tool used to measure feelings of patient-centeredness and empathy within the clinical encounter (Figure 1). The CARE survey was developed and validated at the University of Glasgow to produce a measure that was meaningful to patients regardless of socioeconomic status. The measure is currently used as an appraisal of general practitioners in Scotland and the UK and is included in the National Quality Measures Clearing House in the US. The CARE survey is also used to assess medical student communication and empathy in multiple institutions across the US. ${ }^{11}$ This tool has previously been used to assess student communication and empathy; however, there has never been a study specifically examining the use of the CARE survey in Spanish-speaking patients. Therefore, this study accounts for both the content and construct validity of the Spanish version of the CARE survey (this will be addressed later).

The CARE survey is scored in the following way: 50 items should be collected in order to use the survey as a measurement tool. ${ }^{2}$ Each of the ten items on the survey are scored using the following 5-point Likert scale: "poor" $=1$, "fair" =2, "good" =3, "very good" $=4$, and "excellent" $=5$. Numeric responses from all the ten items are then added together yielding a sum score (maximum possible score of 50 and a minimum possible score of 10). Up to two missing or "not applicable" responses are allowed and these answers are replaced with the average score for the other items. If a survey contains more than two missing or "not applicable" responses, it is not used in the analysis. ${ }^{12}$ The developers of the CARE measure have additionally collected data from general practitioners in Scotland to calculate a mean CARE score of 43 and generate "normative values" for comparison. ${ }^{13}$

\section{Participants}

This Pilot study received prior approval from the Virginia Commonwealth University Institutional Review Board. Study participants were informed that the survey was for research purposes, that it was not mandatory, that it was anonymous, and that completion of the survey would not affect the provision of healthcare. Patients were not required to participate. Completion of the survey was considered as consent. CARE surveys were administered anonymously and voluntarily during patient encounters at CrossOver Ministry Healthcare Clinic (CrossOver) weekly student-led teaching clinic sessions held during both the fall and spring semesters at CrossOver, a free clinic in Richmond, VA, that serves patients who are at or below $200 \%$ of the poverty level. The student-led teaching clinic pairs pharmacy and medical students, along with medical interpreters or medical interpreter students together to lead primary care patient visits at CrossOver, with pharmacy- and medicine-attending faculty supervising all encounters. During orientation, students receive specific training on working with medical interpreters, accessing resources for the uninsured, and provider-empathy and patient-centeredness. Within this model, a patient visit may 
I. Please rate the following statements about today's consultation. Please tick one box for each statement

and answer every statement.

How was the doctor at...

Poor Fair Good Very Excellent Does good not apply

1) Making you feel at ease...

(being friendly and warm toward you,

treating you with respect; not cold or abrupt)

2) Letting you tell your "story"...

(giving you time to fully describe your illness in

your own words; not interrupting or diverting you)

3) Really listening...

(paying close attention to what you were saying; not

looking at the notes or computer as you were talking)

4) Being interested in you as a whole person...

(asking/knowing relevant details about your life,

your situation; not treating you as just a number)

5) Fully understanding your concerns...

(communicating that he/she had accurately understood

your concerns; not overlooking or dismissing anything)

6) Showing care and compassion...

(seeming genuinely concerned, connecting with you on a

human level; not being indifferent or "detached" )

7) Being positive...

(having a positive approach and a positive attitude;

being honest but not negative about your problems)

8) Explaining things clearly...

(fully answering your questions, explaining clearly,

giving you adequate information; not being vague)

9) Helping you to take control...

(exploring with you what you can do to improve your

health yourself; encouraging rather than "lecturing" you)

10) Making a plan of action with you...

(discussing the options, involving you in decisions as

much as you want to be involved; not ignoring your views)

Figure I The Consultation and Relational Empathy (CARE) measure: English.

last an hour or more, as the student pair conducts the patient interview and physical examination, presents an assessment and treatment plan to the precepting faculty member, who then visits together with the patient and team before deciding on a final plan of action.

\section{Content validity}

The original CARE survey is not available in Spanish, therefore, two medically trained Spanish interpreters translated the survey for use in this project. Two native Spanish-speaking staff members at CrossOver then reviewed the survey and made recommendations based on pilot test of the survey with several Spanish-speaking patients. This research was reviewed by the Virginia Commonwealth University Institutional Review Board and qualified for exemption.

Construct validity was measured for the CARE Spanish survey as well using a Cronbach alpha $=0.974$, thus a strong intercorrelation between the items on the CARE Spanish survey.

\section{Statistical analysis}

SPSS $^{\circledR}$, version 16.0 (SPSS Inc., Chicago, IL, USA), was used for all statistical analyses. Descriptive statistics, including medians and interquartile ranges (IQRs) were calculated for the CARE surveys. Because sample sizes were not equal and 
the data were left-skewed, the nonparametric Mann-Whitney $U$ test was performed to explore differences in scores between English- and Spanish-speaking patients. Mean values were calculated for each questionnaire item in each language group for item-ranking purposes.

\section{Results}

A total of 151 surveys were collected, and 149 surveys were included in the analysis, 101 were English speaking and 48 Spanish speaking. Two surveys were not included because they contained more than two missing or "not applicable" responses. Two surveys were corrected with average scores for missing or "not applicable" responses in accordance with the validation study scoring instructions. The distribution of surveys from English- and Spanish-speaking patients during the fall and spring semesters can be found in Table 1.

The overall median score for the entire sample was 50.00, with an IQR of 40.00-50.00. There was a statistically significant difference in scores $(P=0.020)$, with English-speaking patients scoring higher (median [IQR] 50.00 [41.50, 50.00]) than Spanish-speaking patients (median [IQR] 47.50 [38.25, 50.00]).

For each questionnaire item, the minimum and maximum values were 2.00 and 5.00, respectively, with median of 5.00. Table 2 shows the mean for each question of the English- and Spanish-speaking patients. Both groups scored question 8 highest, with English-speaking patients scoring questions 7 and 6 next highest, and Spanish-speaking patients scoring questions 6 and 9 next highest. For English-speaking patients, questions 1,2, and 5 were scored lowest, while for Spanish-speaking

Table I Distribution of CARE surveys

\begin{tabular}{llll}
\hline Language & Fall & Spring & Total \\
\hline English & 42 & 59 & 101 \\
Spanish & 20 & 28 & 48 \\
Total & 62 & 87 & \\
\hline
\end{tabular}

Abbreviation: CARE, Consultation and Relational Empathy.

Table 2 Mean scores for each question by English- and Spanishspeaking patients

\begin{tabular}{lll}
\hline Question* & Mean score (English) & Mean score (Spanish) \\
\hline 1 & 4.57 & 4.38 \\
2 & 4.60 & 4.27 \\
3 & 4.61 & 4.40 \\
4 & 4.58 & 4.38 \\
5 & 4.59 & 4.31 \\
6 & 4.64 & 4.42 \\
7 & 4.64 & 4.33 \\
8 & 4.65 & 4.46 \\
9 & 4.61 & 4.42 \\
10 & 4.61 & 4.40 \\
\hline
\end{tabular}

Note: Refer to Figure I for the questions. patients, the three lowest scored questions were 2, 5, and 7. It is interesting to note that question 7 was scored as one of the highest questions by English-speaking patients but one of the lowest by Spanish-speaking patients. While mean scores were lower on all items for Spanish speakers compared to English speakers, only questions 2 and 7 were statistically significant when item distributions were compared by Wilcoxon signedrank test $(P=0.021$ and $P=0.040$, respectively).

\section{Discussion}

This observational study examined feelings of patientcenteredness and empathy experienced by English- and Spanish-speaking patients seen at the CrossOver student-led teaching clinic over two semesters. Overall, mean scores for both English- and Spanish-speaking patients for the entire year were above the reported normative average of 43 for general practitioners. ${ }^{13}$ Median survey scores from English-speaking patients fell within the 95th percentile, while those from Spanish-speaking patients fell within the 90th percentile. These results are consistent with previous studies that have found that through active student participation, patients gain a better understanding of their disease and are more satisfied that their needs have been met. ${ }^{14}$ Students engaged in the student-led teaching clinic at CrossOver additionally received specific training during orientation focused on patient-centeredness and provider empathy, which may have contributed to higher levels of patient satisfaction. The ability to spend more time with each patient in a multidisciplinary, student-led environment is absent in most general practices and may contribute to higher patient satisfaction in terms of empathy and patientcenteredness compared to normative values, despite the increased visit length experienced in this and other studentled teaching clinics.

These results indicate overall satisfaction with care provided at this clinic in terms of empathy and patient-centeredness; however, disparities did exist, between English- and Spanish-speaking patients. All individual survey questions were ranked lower by Spanish-speaking patients, with two questions (2 and 7) significantly lower. Question 2 asks if patients were able to "tell their story" by telling about the illness in their own words without being diverted, and questions 7 asks if patients felt the team remained "positive" by having a positive attitude and approach and were honest and not negative about problems. Although the result of the CARE survey in Spanish speakers does not exist, previous studies have found that Spanish-speaking patients are less satisfied with health care overall and less satisfied with the provider listening and amount of time spent with the provider. ${ }^{7}$ Research 
suggests that provider-patient communication difficulties may play a significant role in this. ${ }^{6}$ Despite using interpreters, there may also have been a cultural barrier in students being able to consistently display positivity regarding illness, as this was the first time that most of the students were working with interpreters and non-English-speaking patients.

\section{Limitations}

Limitations to this analysis exist. First, a standard survey collection method was not utilized; rather, each team of student providers was responsible for requesting the completion of the survey. This situation may have led to bias in patient responses. Further, sample sizes $<50$ may have limited the statistical analysis, as 50 surveys should be collected in order to use the survey as a measurement tool. ${ }^{2}$ Unfortunately, due to the lower percentage of Spanish-speaking patients seen in this clinic, it was inherently difficult to achieve a larger sample size for that subgroup. Finally, the Spanish version of the CARE survey has not been validated as a measurement tool. This project highlights the need to validate the use of the measurement tool in different languages and cultures.

Despite these limitations, this study illustrates overall patient satisfaction in terms of empathy and patient-centeredness in an outpatient, student-led teaching primary care clinic. The results highlight the importance of training students to be patient-centered, which may allow for increased patient satisfaction with primary care visits. Beyond academia, continuing education focused on improving patient-centeredness and empathy may also benefit general practice settings and improve patient satisfaction. By focusing on provider-patient communication, patient-centeredness and provider empathy, advancements can be made in quality of care.

Results also highlight the continuing need to address the disparities between English- and Spanish-speaking patients with regard to patient satisfaction. While many studies have demonstrated that Spanish-speaking patients have lower satisfaction with health care services, there is a dearth of literature specifically examining feelings of patient-centeredness and empathy in this population. Additional interventions such as education on cultural differences and attitudes toward care are likely necessary to decrease differences between English- and Spanish-speaking patients. Future research focused on such initiatives may help reduce differences in quality of care.

\section{Acknowledgments}

The Virginia Commonwealth University Council for Community Engagement supported the CrossOver-VCU evening clinic through the Community Engagement Grants Program. Tamara Davidson, PharmD and Vilma Seymour translated the Consultation and Relational Empathy Measure. Eleanor Sanchez, Erilisse Deynes, and the staff at the CrossOver helped to administer and collect surveys.

\section{Disclosure}

The authors report no conflicts of interest in this work.

\section{References}

1. Simpson SA, Long JA. Medical student-run health clinics: important contributors to patient care and medical education. J Gen Intern Med. 2007;22(3):352-356.

2. Buchanan D, Witlen R. Balancing ethics and education: ethical management of student-run clinics. J Health Care Poor Underserved. 2006; 17(3):477-485.

3. Zucker J, Lee J, Khokhar M, Schroeder R, Keller S. Measuring and assessing preventive medicine services in a student-run free clinic. $J$ Health Care Poor Underserved. 2013;24(1):344-358.

4. O'Brien M, Shea J. Disparities in patient satisfaction among Hispanics: the role of language preference. J Immigr Minor Health. 2011;13(2):408-412.

5. Welty E, Yeager VA, Ouimet C, Menachemi N. Patient satisfaction among Spanish-speaking patients in a public health setting. J Healthc Qual. 2012;34(5):31-38.

6. Lee LJ, Batal HA, Maselli JH, Kutner JS. Effect of Spanish interpretation method on patient satisfaction in an urban walk-in clinic. J Gen Intern Med. 2002;17(8):641-645.

7. Villani J, Mortensen K. Decomposing the gap in satisfaction with provider communication between English- and Spanish-speaking Hispanic patients. J Immigr Minor Health. 2014;16(2):195-203.

8. Ly DP, Glied SA. Disparities in service quality among insured adult patients seen in physicians' offices. J Gen Intern Med. 2010;25(4);357-362.

9. Institute of Medicine, Crossing the Quality Chasm: A New Health System for the 21st Century. National Academy Press; 2001.

10. Neumann M, Scheffer C, Tauschel D, Lutz G, Wirtz M, Edelhäuser F. Physician empathy: definition, outcome-relevance and its measurement in patient care and medical education. GMS Z Med Ausbild. 2012;29(1):1-21.

11. Mercer SW. The CARE Measure-Summary of Research and Current Use; 2009. Available from: http://www.gla.ac.uk/media/media_141446_en.pdf. Accessed July 1, 2014.

12. Mercer SW, McConnachie A, Maxwell M, Heaney D, Watt GC. Relevance and practical use of the Consultation and Relational Empathy (CARE) measure in general practice. Fam Pract. 2005;22(3): 328-334.

13. Mecer, SW. Normative Values; 2006. Available from: http://www.gla. ac.uk/researchinstitutes/healthwellbeing/research/generalpractice/ caremeasure/values/. Accessed July 1, 2014

14. Scheffer C, Tauschel D, Neumann M, Lutz G, Valk-Draad M, Edelhäuser F. Active student participation may enhance patient centeredness: patients assessments of the clinical education ward for integrative medicine. Evid Based Complement Alternat Med. 2013;2013:743832. 
The Journal of Multidisciplinary Healthcare is an international, peerreviewed open-access journal that aims to represent and publish research in healthcare areas delivered by practitioners of different disciplines. This includes studies and reviews conducted by multidisciplinary teams as well as research which evaluates the results or conduct of such teams or health care processes in general. The journal covers a very wide range of areas and welcomes submissions from practitioners at all levels, from all over the world. The manuscript management system is completely online and includes a very quick and fair peer-review system. Visit http://www.dovepress.com/ testimonials.php to read real quotes from published authors.

Submit your manuscript here: https://www.dovepress.com/journal-of-multidisciplinary-healthcare-journal 